ARTICLE OPEN

\title{
Generalized stacking fault energy surface mismatch and dislocation transformation
}

\author{
Longsheng Feng $\mathbb{D}^{1}$, Michael J. Mills ${ }^{1}$ and Yunzhi Wang $\mathbb{D}^{1 \times}$
}

Even though the fundamental rules governing dislocation activities have been well established in the past century, we report a phenomenon, dislocation transformation, governed by the generalized-stacking-fault energy surface mismatch (GSF mismatch for short) between two co-existing phases. By carrying out ab-initio-informed microscopic phase-field simulations, we demonstrate that the GSF mismatch between a high symmetry matrix phase and a low symmetry precipitate phase can transform an array of identical full dislocations in the matrix into an array of two different types of full dislocations when they shear through the precipitates. The precipitates serve as a passive Shockley partial source, creating new Shockley partial dislocations that are neither the ones from the dissociation of the full dislocation. This phenomenon enriches our fundamental understanding of partial dislocation nucleation and dislocation-precipitate interactions, offering additional opportunities to tailor work-hardening and twinning processes in alloys strengthened by low-symmetry precipitate phases.

npj Computational Materials (2021)7:201 ; https://doi.org/10.1038/s41524-021-00660-z

\section{INTRODUCTION}

The theory of dislocations is an important cornerstone in crystal physics. It was initially proposed to solve the puzzle of why a crystal yields at one-hundredth of its ideal strength ${ }^{1}$, and among the later advancements the mechanisms of dislocation generation and multiplication are probably the most fundamental concepts. During plastic deformation of crystalline materials, dislocations carry the plastic flow by multiplying themselves through mechanisms like the Frank-Read source ${ }^{2}$ and double cross slip ${ }^{3}$, leading to a massive dislocation population explosion. Their mutual interactions as well as interactions with other extended defects present in the crystals generate a rich variety of deformation microstructures that underpin all the important mechanical properties including yield strength, work-hardening ability, ultimate strength, ductility, fracture toughness, creep resistance, etc. There are some longstanding consensuses on dislocation nucleation, multiplication, and interaction with other extended defects in a crystal. For instance, when a dislocation multiplies through a Frank-Read source, where a single full dislocation segment is pinned at two ends, it continuously generates dislocations of the same Burgers vector and on the same slip plane as that of the Frank-Read source segment ${ }^{2}$. When dislocations meet homo-phase interfaces such as grain/twin boundaries in polycrystalline materials, they could be absorbed into the interfaces or transmitted to the other side of the interfaces, the latter is referred to as slip transmission or transmutation ${ }^{4}$, where the transmitted dislocations can have different slip planes and Burgers vectors. The mechanisms of slip transmission or transmutation underpin grain boundary strengthening. For precipitate-strengthened alloys, dislocations either shear trough or loop around the precipitates. Both shearing and looping mechanisms have been utilized widely in the design of commercial alloys where significant amounts of strength arise from dislocationprecipitate interactions. Dislocations can also climb-bypass precipitates when temperature is high enough to activate vacancy diffusion. Despite the complexity in dislocation-precipitate interactions, it is always assumed that the dislocations approaching and leaving the precipitates are the same.
In this study, we demonstrate a concept referred to as generalized stacking fault energy surface mismatch (will be referred to as GSF mismatch hereafter) between matrix and precipitate phases. Because of such a GSF mismatch, an array of partial dislocations can nucleate in the low-symmetry precipitate phase when an array of full dislocations with the same Burgers vector (like those generated by a Frank-Read source) shear through the precipitates, leading to a change in the dislocation content when they exit the precipitates. The nucleated Shockley partial can also move towards the entrance side of the precipitate as the partial loop expands into the matrix under the applied stress. We refer to this phenomenon as dislocation transformation to distinguish it from dislocation transmutation during slip transmission across a bicrystal interface defined in the literature ${ }^{4}$, where the same dislocations have not actually transmitted, but the action of slip has been transmitted. The results uncovered in this work provide an essential piece in understanding dislocationprecipitation interaction and adds another perspective on dislocation generation and multiplications.

\section{RESULTS \\ GSF mismatch}

An important class of structural materials for high-temperature applications, i.e., the superalloys, relies on strengthening by precipitates of ordered intermetallic phases that are stable at high temperatures. When an ordered phase precipitates out of a disordered parent phase, it may break only the translational symmetry or both the translational and the point symmetries. Take the $\mathrm{Ni}_{3} \mathrm{X}$-type ordered precipitate phase family in Ni-base superalloys as an example, which include $\mathrm{L}_{2}, \mathrm{DO}_{\mathrm{a}}, \mathrm{DO}_{19}, \mathrm{DO}_{22}$, and $\mathrm{DO}_{24}{ }^{5-8}$. The matrix FCC phase (space group, $\mathrm{Fm} \overline{3} \mathrm{~m}$ ) has a point group $m \overline{3} m$, while the $L 1_{2}$ precipitate phase (space group, $P m \overline{3} m$ ) has the same point group $(m \overline{3} m)$, but loses the $a / 2<110>$ translational symmetry, which results in four different anti-phase domains. The $\mathrm{DO}_{22}$ precipitate phase (space group, $\mathrm{I}_{4} / \mathrm{mmm}$ ), on the other hand, breaks both the translational $(a / 2<110>)$ and

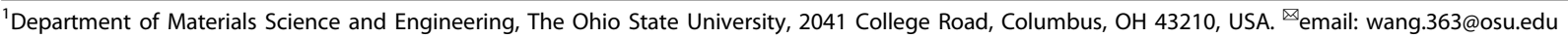


point (3-fold rotational symmetry in $\{111\}$ planes) symmetries of the FCC structure. As a result, a displacement vector that ends at a stable equilibrium position in the matrix phase (i.e., local minimum energy location on the generalized stacking fault (GSF) energy surface) may not be at a stable equilibrium position anymore in the precipitate phase (see, e.g., Fig. 1). Since the IN718 Ni-base superalloy is strengthened by both $\mathrm{L1}_{2}\left(\gamma^{\prime}\right)$ and $\mathrm{DO}_{22}\left(\gamma^{\prime \prime}\right)$ phases, we will use it as an example in our following discussions.

Figure 1 shows the GSF surfaces of the $\{111\}$ slip planes in $\gamma$ (the FCC matrix), $\gamma^{\prime}$ and $\gamma^{\prime \prime}$ phases obtained by ab initio calculations ${ }^{9,10}$. Various stacking faults are labeled on the figure and the Burgers vectors of dislocations are given in the inset. As mentioned earlier, the loss of $a / 2<110>$ translations results in the formation of APB in $\gamma^{\prime}$ and $\gamma^{\prime \prime}$. However, the three-fold point symmetry of the $\{111\}$ plane in $\gamma$ is preserved in $\gamma^{\prime}$ but broken in $\gamma^{\prime \prime}$. The differences in the GSF surfaces of these three phases are shown in Fig. $1 \mathrm{~b}, \mathrm{~d}$ and $\mathrm{f}$ by plotting the energy landscapes along the $[\overline{1} 2 \overline{1}]$ direction on the GSF surfaces (red dash arrows in Fig. 1a, c, e). It is readily seen that all the stable equilibria in $\gamma$ are preserved for the same displacements in $\gamma^{\prime}$, but not in $\gamma^{\prime \prime}$. For example, the $a / 2[\overline{1} 01]$ displacement (solid black arrows in Fig. 1a, c, e) restores the FCC structure in $\gamma$ and creates an APB in $\gamma^{\prime}$, both of which are stable equilibrium positions on the GSF surfaces. However, the same displacement in $\gamma^{\prime \prime}$; ends at an APB-like fault position, which is an unstable position on the GSF surface. These positions are indicated by the second vertical red dash line in Fig. $1 \mathrm{~b}, \mathrm{~d}$, e. A similar observation can be made for the displacement of $a / 6[1 \overline{2} 1]$ (the solid orange arrows in Fig. 1a, c, e), which leads to the formation of an intrinsic stacking fault (ISF) in $\gamma$, a CSF in $\gamma^{\prime}$ and a CSF in $\gamma^{\prime \prime}$ (see the positions indicated by the third vertical red line in Fig. $1 b, d, e)$. Since the term "unstable stacking fault" has been used to represent energy maxima on the GSF surface, the unstable

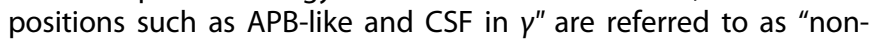
equilibrium stacking fault" because they are not equilibrium positions (i.e., local minima or maxima) on the GSF surface. Note that not all the stable equilibrium positions in $\gamma$ are disrupted by the symmetry breaking. For example, the displacement of $a / 3[\overline{112}]$ (the solid purple arrows in Fig. 1a, c, e) in all three phases corresponds to local minima (ISF in $\gamma$ and $\gamma^{\prime \prime}$ and SISF in $\gamma^{\prime}$ ). This is indicated by the first vertical red line in Fig. $1 b, d$, e.

Let us refer to these differences in the stable equilibrium positions on the GSF surfaces of the matrix and precipitate phases caused by symmetry breaking as GSF mismatch (in an analogy to lattice or modulus mismatch between two co-existing phases). If the GSF mismatch is created by the breaking of a translational symmetry such as the $a / 2<110\rangle$ translations, then all displacements leading to stable equilibrium positions on the GSF surface of the matrix phase will still end at stable equilibrium positions in the precipitate phase. In this case, the GSF mismatch is characterized by the difference in planar faults (e.g., an FCC structure in $\gamma$ and an APB in $\gamma^{\prime}$ ). If the GSF mismatch is caused by the loss of a point symmetry such as the 3 -fold axis in $\{111\}$ planes, then not all stable equilibrium positions on the GSF surface of the matrix phase will be preserved in the precipitate phase. In this
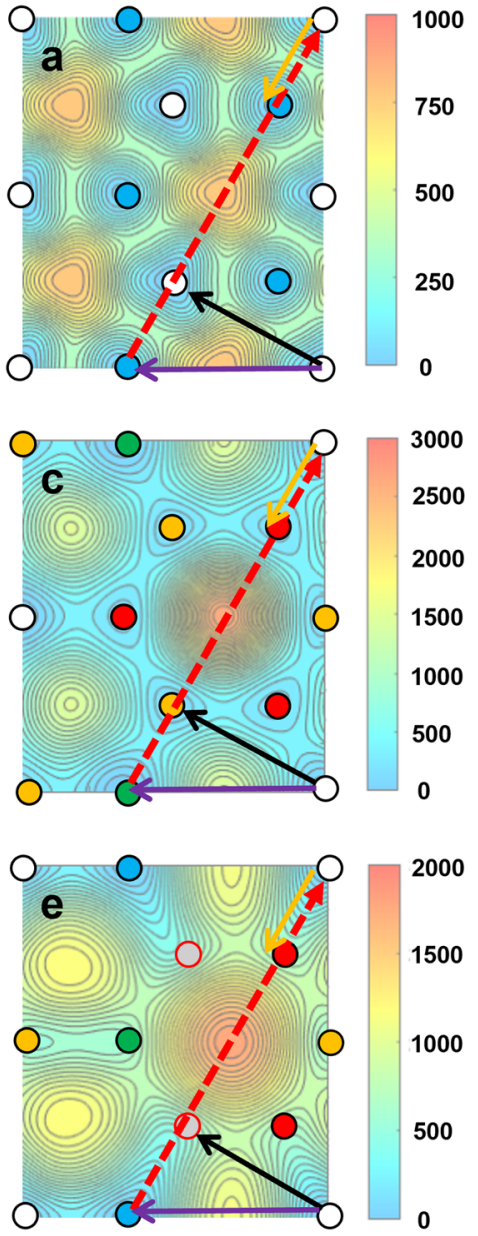

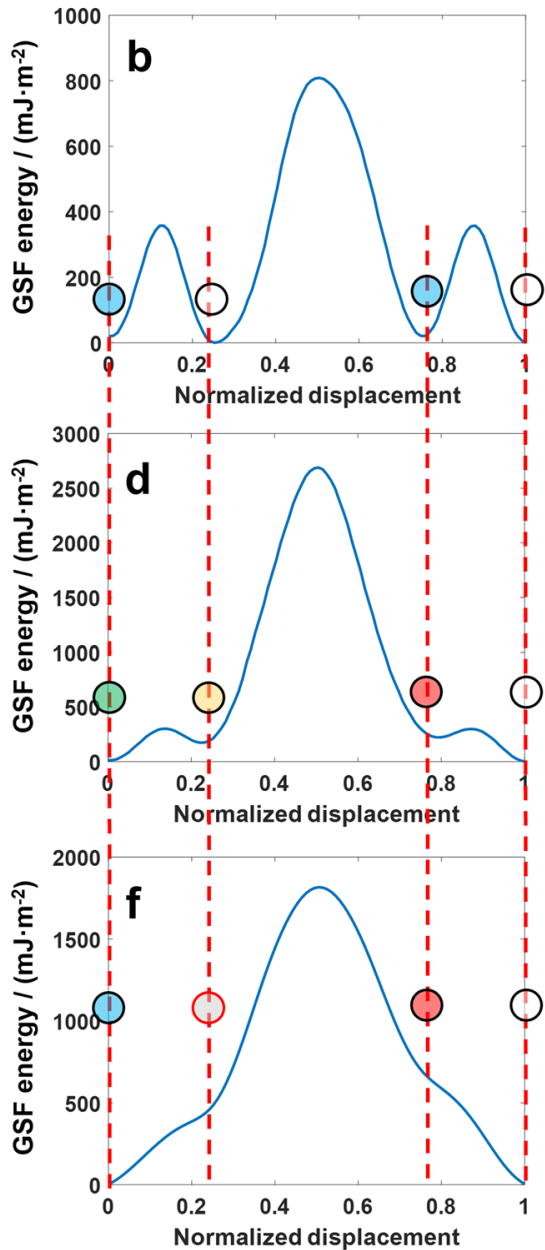

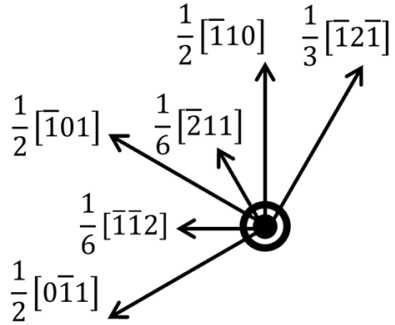

No Fault (NF)

CSF

$\gamma^{\prime}: 220 \mathrm{~mJ} \cdot \mathrm{m}^{-2}$

$\gamma^{\prime \prime}: 698 \mathrm{~mJ} \cdot \mathrm{m}^{-2}$

( $\mathrm{APB}$

$\gamma^{\prime}: 172 \mathrm{~mJ} \cdot \mathrm{m}^{-2}$

$\gamma^{\prime \prime}: 592 \mathrm{~mJ} \cdot \mathrm{m}^{-2}$

O ISF

$\gamma: 20 \mathrm{~mJ} \cdot \mathrm{m}^{-2}$

$\gamma^{\prime \prime}: 2.3 \mathrm{~mJ} \cdot \mathrm{m}^{-2}$

SISF

$\gamma^{\prime}: 13 \mathrm{~mJ} \cdot \mathrm{m}^{-2}$

$\gamma^{\prime \prime}: 614 \mathrm{~mJ} \cdot \mathrm{m}^{-2}$

APB-like

$\gamma^{\prime \prime}: 482 \mathrm{~mJ} \cdot \mathrm{m}^{-2}$

Fig. 1 GSF analysis of $\gamma, \gamma^{\prime}$ and $\boldsymbol{\gamma}^{\prime \prime}$ phases. GSF surface of \{111\} plane of a $\gamma, \mathbf{c} \gamma^{\prime}$, and e $\gamma^{\prime \prime}$ phases and the energy landscape along the $[\overline{1} 2 \overline{1}]$ direction of $\mathbf{b} \gamma, \mathbf{d} \gamma^{\prime}$, and $\mathbf{f} \gamma^{\prime \prime}$ phases. The no fault (NF) structure is the stacking-fault-free structure in all three phases. The legends for GSF contour plots in $(\mathbf{a}, \mathbf{c}, \mathbf{e})$ have units of $\mathrm{mJ} \mathrm{m}^{-2}$. The same unit is applied to all GSF contour plots. 
case, the GSF mismatch is characterized by the loss of stable equilibrium positions (e.g., the APB-like fault in $\gamma^{\prime \prime}$ ). The former GSF mismatch leads to ordering strengthening and has been studied extensively and utilized widely in high-temperature alloy design $^{11-14}$. However, the latter GSF mismatch has not been widely recognized and utilized judiciously in alloy design. Since such a mismatch leads to the loss of equilibrium positions and a shear cannot stop at an unstable position, the displacement must find a nearby local minimum position to reach the stable mechanical equilibrium. What would happen if such a low symmetry precipitate phase is continuously sheared by a stream of identical dislocations generated by a Frank-Read source operating in the matrix phase? To answer this question, we use the microscopic phase field model of dislocations ${ }^{15-22}$ with $a b$ initio calculated GSF surfaces to study the shearing process of $\gamma^{\prime \prime}$ precipitates that are coherently embedded in a $\gamma$ matrix. For simplicity, the multicomponent superalloy is represented by a binary $\mathrm{Ni}$-Nb system. This simplification will not make qualitative changes to our findings.

\section{Dislocation transformation}

The symmetry breaking during the $\mathrm{FCC}$ to $\mathrm{DO}_{22}$ phase transformation leads to three different correspondence variants of the $\gamma^{\prime \prime}$ phase $^{23}$. For simplicity, we start our simulations with an array of identical $\gamma^{\prime \prime}$ particles of the same variant, which are evenly spaced and have an elliptical shape with a major axis of $38 \mathrm{~nm}$ and a minor axis of $13 \mathrm{~nm}$ on the (111) cross-section plane (slip plane) ${ }^{24}$ in the middle of a periodically repeating simulation cell (Fig. 2). Consecutive CA (Burgers vector $a / 2[0 \overline{1} 1]$ ) dislocations are sent from the bottom of the simulation cell (e.g., from a CA Frank-Read source in the matrix). The dislocations move upward under an applied shear stress of $800 \mathrm{MPa}$ along $[\overline{11} 2]$, which is high enough to push the dislocations passing through the precipitate array.

Figure 2 shows the interactions between 3 consecutive CA dislocations and the $\gamma$ " precipitates, where we plot the GSF energy of the system. Pixels with energy values smaller than $100 \mathrm{~mJ} / \mathrm{m}^{2}$ is set to be transparent to highlight the dislocation line (i.e., the dislocation core) and high energy faults. The 1st CA (first row, Frames 1-6) cuts into the precipitates and creates an APB-like fault at the tips (Frame 2). Since the APB-like fault is a non-equilibrium stacking fault, an ISF island (transparent in the plot), i.e., a Shockley partial dislocation loop, is nucleated simultaneously in each of the $\gamma^{\prime \prime}$ precipitates (Frame 3), eliminating the APB-like fault and bringing the displacement to the adjacent local minimum (see the yellow and gray arrows in Fig. 3a). The vector connecting the APBlike fault and the ISF on the GSF surface is the newly generated Shockley partial dislocation $\delta \mathrm{B}(a / 6[\overline{2} 11])$, as indicated by the gray arrow in Fig. 3a). The bottom insets in Frame 2 and 3 shows the dislocation lines of each frame from the displacement field analysis (taken directly from Frame 2 and Frame 3 of Supplementary Fig. 1, see details of the displacement analysis in
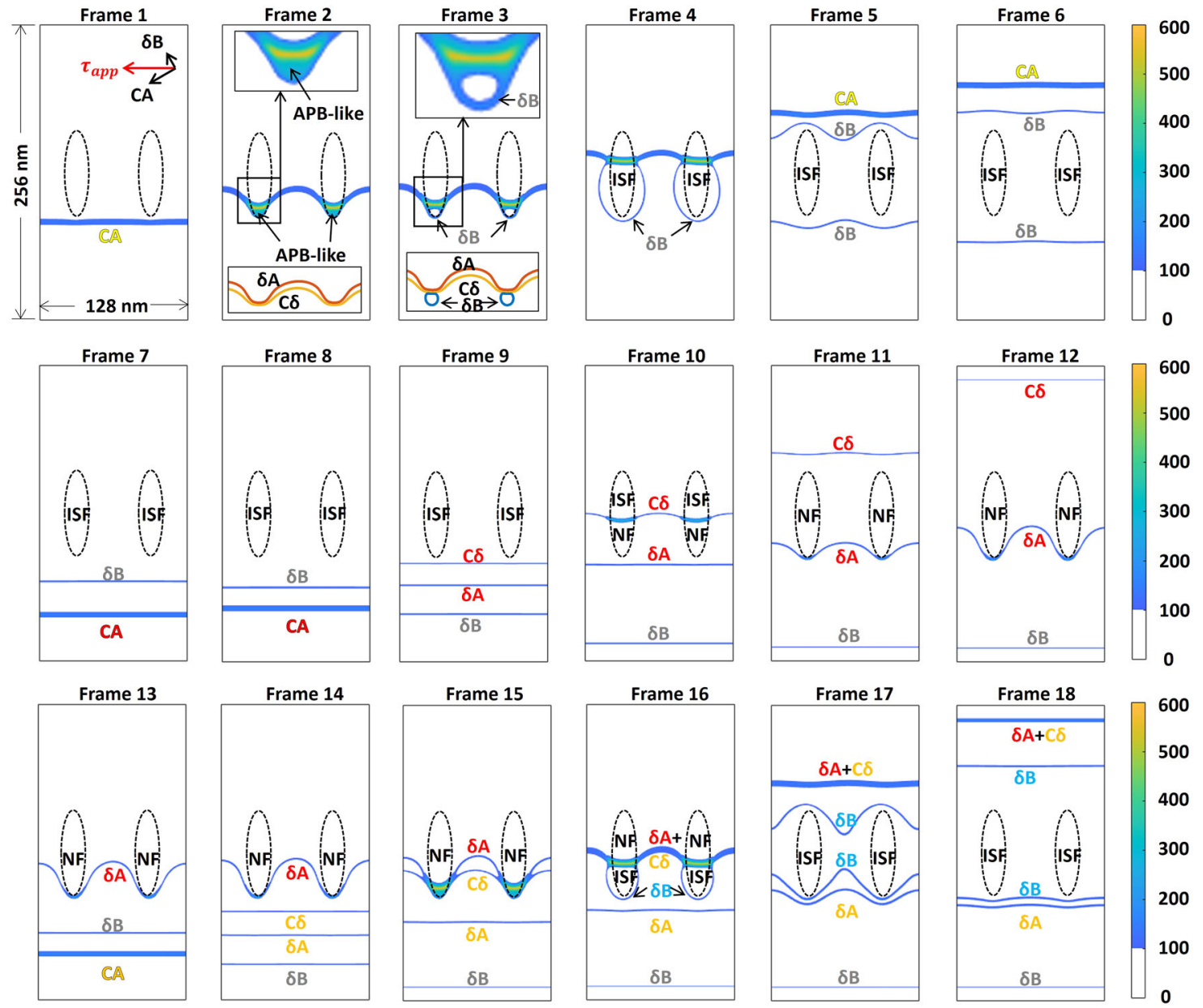

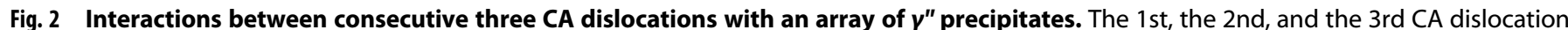
interacting with $\gamma^{\prime \prime}$ precipitates are shown in Frames 1-6, Frames 7-12 and Frames 13-18 respectively. The downward moving $\delta B$ partial on the entrance side of the precipitate array at the Frame 6 and Frame 12 have been shifted upward in Frame 7 and Frame 13, respectively, in order to introduce the 2nd and 3rd upcoming CA dislocations from the bottom of the simulation cell. The dislocations on the exit side of the precipitate array at Frame 6 and Frame 12 have been documented in Fig. 3c and cleared in Frame 7 and Frame 13 for better visualization, respectively. The legends have units of $\mathrm{mJ} \mathrm{m}^{-2}$. 

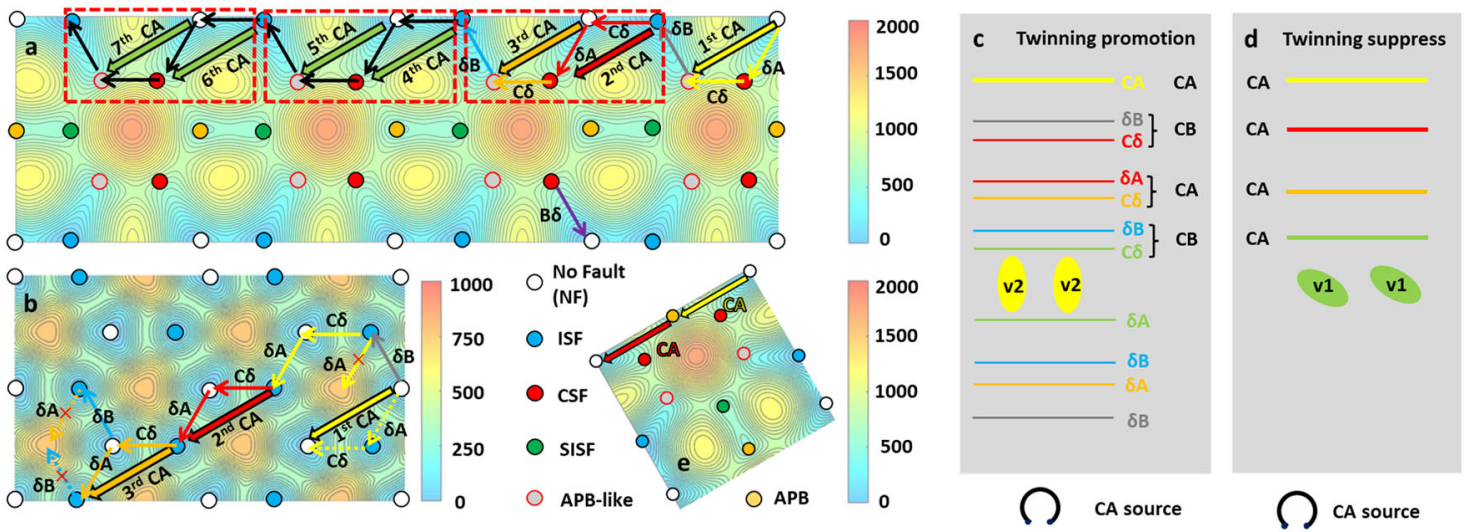

Fig. 3 Deformation pathways and dislocation content in the dislocation transformation process. Deformation pathways of a $\gamma^{\prime \prime}$ and $\mathbf{b} \gamma$ phases shown in Fig. 2. c Summary of dislocations in Fig. 2. d Schematic precipitate microstructure suppressing twinning in $\gamma^{\prime \prime}$-strengthened IN718 Ni-base superalloys. e The deformation pathway for the precipitate microstructure in d. The variant promoting twinning when CA dislocation is active is named $\mathrm{v} 2$ while the one suppressing twinning is named $\mathrm{v} 1$ to distinguish one from the other.

Supplementary Note). Note that the newly generated Shockley partial $\delta B$ is not part of $C A$. The $\delta B$ dislocation loops expand into the $\gamma$ matrix under the applied shear stress, coalesce with each other and split into two dislocation lines with opposite line directions, with one moving upward and the other moving downward (Frames 3-6). The final deformation microstructure (i.e., dislocation configuration) is a $C A+\delta B$ dislocation group moving upward at the exit side and a single $\delta B$ Shockley partial moving downward at the entrance side of the precipitate array (Frame 6).

Frames 7-12 of Fig. 2 show the passing of the 2nd CA through the new dislocation generated and the $\gamma^{\prime \prime}$ precipitates. It first encounters the newly generated $\delta B$ partial that is moving in the opposite direction. Since the Burgers vectors of $\delta B$ and $C A$ dislocations are perpendicular to each other, there is no interaction between them. However, after $C A$ passes through $\delta B$, the dissociation sequence of $C A$ changes from $\delta A$ (leading) $+C \delta$ (trailing) to $C \delta$ (leading) $+\delta A$ (trailing) (Frames 7-9). This process is illustrated in Fig. $3 b$, with the dash and solid yellow arrows indicating the dissociation sequence before and after CA passes $\delta B$, respectively. When the leading partial $C \delta$ encounters the $\gamma^{\prime \prime}$ precipitates, it cuts into them, eliminating the ISF and restoring the $\mathrm{DO}_{22}$ structure of $\gamma^{\prime \prime}$ (Frame 10), while the trailing $\delta \mathrm{A}$ partial stops at the $\gamma / \gamma^{\prime \prime}$ interface (Frames 11 and 12). The deformation pathway is plotted on the GSF surface in Fig. 3a using the red arrows. The final microstructure is $C \delta$ partial moving upward and $\delta A$ partial stuck at the $\gamma / \gamma^{\prime \prime}$ interface, while the $\delta B$ partial continuing moving downward (Frame 12).

Frames 13-18 of Fig. 2 show the passing of the 3rd CA through the $\delta B$ partial generated and the $\gamma^{\prime \prime}$ precipitates. Similar to the 2 nd $C A$, the $3 r d C A$ reverses its dissociation sequence when it passes the downward moving $\delta B$ (Frames 13 and 14). Then the shearing process of the $\gamma^{\prime \prime}$ precipitates repeats what was observed in the interactions between the $1^{\text {st }} \mathrm{CA}$ and the precipitates. As a result, another remnant Shockley partial $\delta B$ is nucleated and expands into the $\gamma$ matrix (Frames 16-18). The deformation pathway is also plotted in Fig. 3a with orange arrows. Unlike the shearing process of the $\gamma^{\prime \prime}$ precipitates by the $1 \mathrm{st} C A$, though, the trailing $\delta A$ of the $3 r d C A$ does not participate in the creation of the 2 nd $\delta B$ because the trailing $\delta A$ of the 2nd CA (stuck at the $\gamma / \gamma^{\prime \prime}$ interface (Frames 11 and 12)) pairs with the leading partial $C \delta$ of the 3rd CA and forms a $\delta A+C \delta$ (i.e., normally dissociated $C A$ ) dislocation configuration. This trailing $\delta A$ of the 3rd CA stops the downward movement of the newly nucleated $\delta B$ in the $\gamma$ matrix because if they bypass each other (see the dash blue or orange arrows in Fig. 3b), a fault with the maximum energy on the GSF surface will be created. Since the applied stress has equal resolved shear stress on the two partials, they stay as a stationary dislocation pair in the $\gamma$ matrix
Table 1. Total energies of faults created for the distinctive pathways of the $\gamma^{\prime \prime}$ phase.

\begin{tabular}{ll}
\hline Pathway & Total fault energy $/\left(\mathrm{mJ} \mathrm{m}^{-2}\right)$ \\
\hline $\mathrm{NF} \rightarrow \mathrm{CSF} \rightarrow \mathrm{APB}-$ like $\rightarrow \mathrm{NF}$ & 1180 \\
$\mathrm{NF} \rightarrow \mathrm{CSF} \rightarrow \mathrm{APB} \rightarrow \mathrm{CSF} \rightarrow \mathrm{NF}$ & 1988 \\
\hline
\end{tabular}

(Frame 18). The final deformation microstructure after passing of the 3rd CA dislocation is $\delta A+C \delta+\delta B$ moving upward on the exist side and $\delta B+\delta A$ stuck in the $\gamma$ matrix on the entrance side.

When more CA dislocations pass through the $\gamma^{\prime \prime}$ precipitates, the deformation pathway on the GSF surface shown in the red dash box on the right in Fig. 3a starts to repeat itself, as outlined in the middle and left boxes. The reoccurring pattern indicates that there is one new $\delta B$ Shockley partial generated for every two $C A$ dislocations passing through the $\gamma^{\prime \prime}$ precipitate array (Table 1). The matrix dislocation array now is able to produce an array of Shockley partial dislocations that do not originate from the matrix dislocations, i.e., a partial dislocation source. It must be emphasized that the mechanism for constant Shockley partial dislocation generation demonstrated in the current study is not related to and does not dependent on the dissociation of full dislocations but associated with the GSF mismatch between the precipitate and matrix phases, i.e., the partial dislocations generated by this mechanism are none of those that could be generated by the dissociation of the full dislocation. This partial dislocation source is also different from the regular dislocation sources that it can operate on its own. This partial dislocation source is only activated when a full dislocation source is nearby and hence, it is a passive source. It is also different from the regular dislocation multiplication mechanism like the Frank-Read source because multiplication of dislocations always creates full dislocations with identical Burgers vectors. Because the precipitates act as a dislocation source, the precipitate shearing process does not satisfy conservation of Burgers vector anymore.

In terms of the type of dislocations on both the entrance and exit sides of the precipitate array, a summary is presented in Fig. $3 c$ for the first four CA dislocations (the color of each dislocation matches that in Figs. 2 and 3a). Before the shearing events, only $C A$ dislocations exist in the system on the entrance side of the precipitates (or $\delta A+C \delta$ when CA dissociates). After shearing, on the entrance side, one Shockley partial $\delta B$ (gray) is generated, which is moving away from the precipitates, and one stationary $\delta B / \delta A$ Shockley partial pair appears in the matrix (i.e., $C A+\delta B+$ $\delta A)$. On the exit side, besides the original CA $(a / 2<110>$ type) 
dislocations, a $C A+C B$ dislocation group ( $a / 2<112>$ type) appears in the $\gamma$ matrix. The recurring pattern indicates that every two $C A$ dislocations entering the precipitate will become a $C B+C A$ dislocation group when they exit (the red box in Fig. $3 a$ and $c$ ). Clearly the $\gamma^{\prime}$ precipitates transform the CA dislocations into CA + $C B$ dislocation groups on the exit side and $\delta B / \delta A$ dislocation pairs on the entrance side, which is referred to as dislocation transformation. Note that all these dislocations are on the same (111) slip plane where a CA source is operating.

\section{DISCUSSIONS}

On the entrance side of the precipitate (Fig. 2), the first $\delta B$ is free to move in the matrix and, thus, it will shear/loop other $\gamma^{\prime \prime}$ precipitates of all three variants in its path, independent of whether the ISF can be formed within the precipitate. It will also interact with the $\gamma^{\prime}$ precipitates and will loop around them since the resulting stacking fault in $\gamma^{\prime}$ upon shearing is CSF, which has a high energy and thus difficult to form. If such a Shockley partial exists on the adjacent plane, they could shear the $\gamma^{\prime}$ precipitates together and form an SESF with reordering, which could serve as an embryo of twin ${ }^{25}$. The stationary $\delta A / \delta B$ dislocation pairs on the entrance side can move if the applied stress favors either of them. They may move as pairs, but they will not cross each other as an energy maxima on the GSF will form upon such crossing. If the applied stress favors $\delta B$, the pair will move away from the precipitates and there will be a stream of $\delta B$ partials moving in the opposite direction of the upcoming $C A$ dislocation. If the stress direction favors $\delta A$, the dislocation pairs will move towards the precipitates and the precipitates may be sheared by $\delta B$ again, causing more complicated deformation pathways.

On the exit side of the precipitate, the transformed dislocations $\mathrm{CA}+\mathrm{CB}$ will further shear the rest of the $\gamma^{\prime \prime}$ precipitates in their pathway, but there will be no nucleation of Shockley partials, nor will there be dislocation transformation for the following shearing event since the entrance dislocation is now already $C A+C B$. But both the dislocation transformation and the partial dislocation source can have interesting effects on deformation of other precipitate phases present and, hence, on the overall mechanical properties. If we assume CA dislocation sources are active on multiple slip planes, on the planes where the $\gamma^{\prime \prime}$ precipitates have been sheared, the transformative process changes the dislocations content on those the slip planes into $C A+C B$ whereas on the planes where the $\gamma^{\prime \prime}$ precipitates haven't been sheared or there are no $\gamma^{\prime \prime}$ precipitates, the dislocation content is still CA. Therefore, the $\gamma^{\prime}$ phase in the system can go through two different deformation pathways depending on whether the dislocation transformation is active on a given slip plane. If the matrix source dislocations directly shear through the $\gamma^{\prime}$ phase, APB shearing (CA $+C A$, orange and green arrows in Fig. 4) is the dominant deformation mechanism for the $\gamma^{\prime}$ phase. But with the dislocation transformation mechanism, SISF will be formed in the $\gamma^{\prime}$ phase $(C A+C B$, yellow and blue arrows in Fig. 4), which will never occur in the $\gamma^{\prime}$-strengthened alloys with just CA dislocations active in the system. The mechanism-based crystal plasticity model therefore must take into account different deformation mechanisms of the $\gamma^{\prime}$ phase even though only one slip system is active.

Not all matrix dislocations will lead to dislocation transformation in this system. Some of the stable faults of the matrix phase are preserved in the $\gamma^{\prime \prime}$ phase, such as the APB created by $a / 2[\overline{1} 10]$ (Fig. 1). Therefore, one could use stress-aging 24,26 to produce variants that do not transform the source dislocations under the deformation conditions (e.g., Fig. 3d) and the corresponding deformation pathway would be APB ribbon shearing shown in Fig. 3e. Comparing deformation microstructures in Fig. $3 c, d$, different mechanical properties can be expected. With the dislocation transformation, even though the source dislocation is $C A$, the effective deformation pathway for $\gamma^{\prime \prime}$ precipitates in Fig. $3 \mathrm{C}$ is $\mathrm{CA}+\mathrm{CB}$, which can be simplified into $\mathrm{NF} \rightarrow \mathrm{CSF} \rightarrow \mathrm{APB}$-like $\rightarrow$ ISF by just counting the stacking faults along the pathway. Without dislocation transformation, the deformation pathway for $\gamma^{\prime \prime}$ precipitates (Fig. 3d) is still CA + CA, which can be simplified into NF $\rightarrow$ CSF $\rightarrow$ APB $\rightarrow$ CSF $\rightarrow$ NF by just counting the stacking faults. The former pathway has a smaller total energy than the latter, indicating that the microstructure in Fig. $3 \mathrm{c}$ would have a lower strength than that in Fig. 3d. On the other hand, the dislocation transformation process generates more dislocations due to the nucleation of new Shockley partial $\delta B$ in the system, indicating a higher work-hardening behavior for the microstructure in Fig. 3c than that in Fig. 3d. Depending on the application, one can design the precipitate microstructure through stressaging to select preferred precipitate variants such that desired properties can be achieved.

The partial dislocation source associated with the dislocation transformation could be responsible for the extensive microtwinning observed in the deformation microstructures of alloy IN718 27,28 . Conventional Shockley partial dislocation sources for twinning in FCC is categorized into two types, glide and prismatic ${ }^{29}$, both coming from the dissociation of a full dislocation but different on whether the Burgers vector of the full dislocation lies on the twinning plane. Moreover, twinning propensity greatly depends on the stacking fault energy. A low stacking fault energy alloy tends to have more twins while a high stacking fault energy

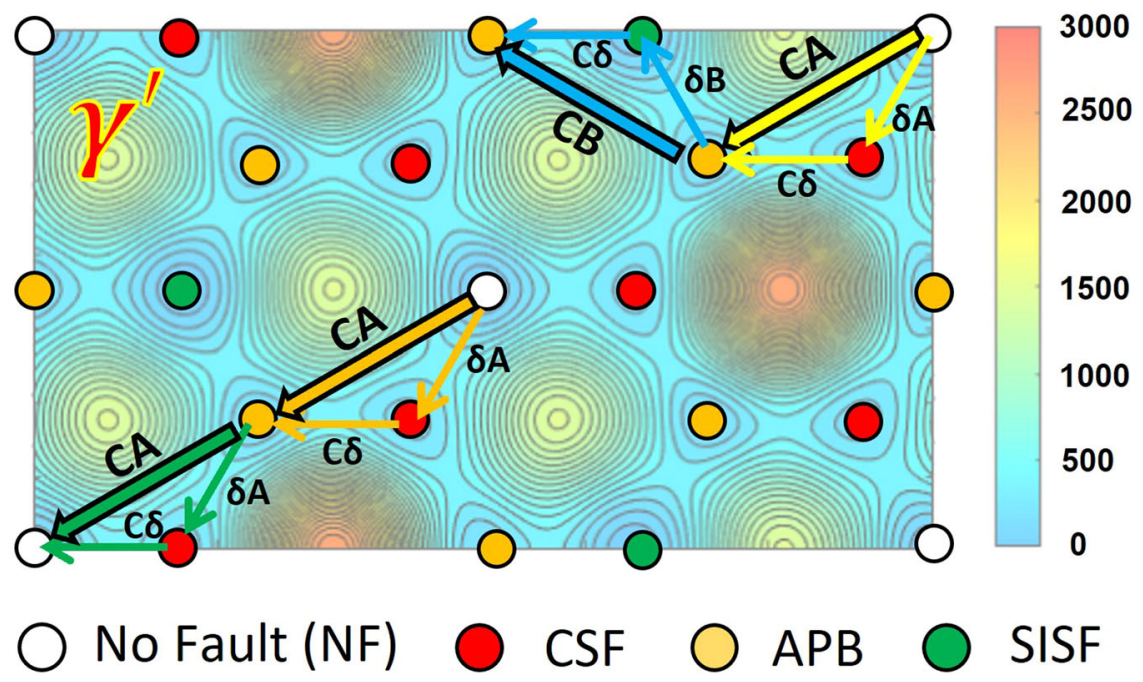

Fig. 4 Deformation pathways of $C A+C A$ and $C A+C B$ dislocation groups in the $\gamma^{\prime}$ phase. 
alloy tends not to, because low stacking fault energy corresponds to wide dissociation of the full dislocations, i.e., more Shockley partials. But the current mechanism to generate Shockley partials for twinning does not depend on the dissociation of the full dislocation, nor does it depend on the stacking fault energy. The ability of generating a stream of partial dislocations resides in the GSF mismatch, which is determined by the crystal structure difference between matrix and precipitate rather than the crystal chemistry of the precipitate phase. This reduces the limitations in alloy design where alloy chemistry is carefully chosen to fulfill multiple objectives. One only needs to consider whether the GSF mismatch exists or not for given crystal structures of matrix and precipitate phases, not the alloy compositions that can be quite sensitive to other properties of the alloy. Similar stress-aging experiment for variant selection can be used to control twinning formation. If twinning is desired, the precipitate microstructure can be stress-aged such that the dislocation-transforming variant is overwhelmingly dominant in the system (e.g., Fig. 3c). The propensity for microtwinning should be significantly reduced in the microstructure shown in Fig. $3 d$ than that shown in Fig. $3 c$ and, therefore, an enhanced creep-resistance could be expected for the microstructures in Fig. 3d because microtwinning has been identified as a weak mode of high temperature creep deformation for single crystals ${ }^{10,26,30}$.

Figure 1 shows two non-equilibrium stacking faults in the $\gamma^{\prime \prime}$ phase, APB-like and CSF. The dislocation transformation mechanism demonstrated above is mediated by the APB-like fault. In principle, the CSF can also transform itself into a stable structure by nucleating a Shockley partial dislocation loop. Moreover, the concept of GSF mismatch is general and thus the conclusions obtained in this study are not limited to this system but applicable to all low-symmetry precipitate-hardened materials where a GSF mismatch is present. Yet, the critical stress required for the dislocation shearing process may depend on model inputs, i.e., the GSF energy surface, the elastic constants and the gradient energy coefficient. Among these input parameters, the GSF energy surfaces, in particular, its symmetry, determines the dislocation transformation mechanisms because it is the GSF mismatch between the precipitate and matrix phases that leads to the dislocation transformation, while the other parameters may influence the quantitative value of the critical stress to push the dislocations through the precipitates.

Note that the dislocation transformation demonstrated in this study is distinctively different from the current understanding of slip transformation across hetero-phase interfaces ${ }^{31-36}$ even though both phenomena cause the change of Burgers vector after interaction with hetero-phase interfaces. Take the interaction between the first CA and the precipitates shown in Fig. 2 as an example. From the slip transmission point of view, the dislocations change the Burgers vector after they enter the precipitate phase, i.e., cross the matrix/precipitate interface from $C A$ to $C A+\delta B$. But there are several crucial differences separating these two phenomena. Due to the change of slip systems during slip transmission, the difference between the entering and exiting dislocations results in a residual Burgers vector deposited at the interface, which is not a lattice vector and thus can only stay at the interface. In dislocation transformation, the difference between the dislocations entering and leaving the $\gamma / \gamma^{\prime \prime}$ interface is a Shockley partial $\delta B$, which is a lattice vector and can move in both matrix and precipitate phases. Therefore in slip transmission, one dislocations enters the interface from one side and a different dislocation comes out on the other side of the interface, whereas in dislocation transformation, one dislocation enters the interface from one side, different dislocations come out on both sides of the interface. Moreover, in slip transmission, a dislocation changes its Burgers vector when transmitted from phase $A$ to phase $B$ through an $A / B$ interface and it will change again when the transmitted dislocation in phase $B$ re-enters phase $A$ across a B/A interface.
Thus, for a multiplayer $A / B$ system, the slip transmission will occur at every $A / B$ and $B / A$ interface. But in dislocation transformation, since the transformed dislocations can also move in the matrix, the dislocation will not change its Burgers vector when it re-enters the matrix, i.e., the change of Burgers vector will only occur at the first matrix/precipitate interface but not precipitate/matrix interface when the transformed dislocation leave the precipitates, nor at all other matrix/precipitate and precipitate/matrix interfaces after the first encounter of the source dislocation and the precipitates.

Another important feature of slip transmission is that it is the shear event that is being transmitted whereas in dislocation transformation the dislocation itself enters the precipitate phase. Because of the discontinuity of slip planes at the interface (either due to the discontinuity of the lattice planes or different slip systems on two sides of the interface), dislocations approaching an interface on one side will be absorbed by the interface and then a new slip event of a different slip system is nucleated on the other side of the interface. While in dislocation transformation, the slip plane continuously extends from matrix phase to precipitate phase such that the dislocation can move through the interface without much resistance. Yet, there are cases where slip planes are continuous across a hetero-phase interface, like multilayer thin films with both phases as FCC and the orientation relationship between the two phases is cube-on-cube (e.g., $\mathrm{Cu} / \mathrm{Ni}$ deposited on (001) planes). But there is no intrinsic crystal symmetry mismatch between the two phases. The distinctive features of the dislocation transformation reside in both continuous slip planes between the two phases involved and the intrinsic crystal symmetry mismatch between them, which is rare in the current study of slip transmission, but widely exist in matrix-precipitate studies. Of course, if one were to make multilayer thin films with the $\gamma$ and $\gamma^{\prime \prime}$ phases, the above dislocation transformation would still occur as a new type of slip transmission. But it is of great interest for the alloy design community to realize the distinctive features of this dislocation transformation phenomena that the precipitation shearing process can be looked at from a slip transmission point view that the slip systems can change upon shearing, which enables using the low-symmetry precipitate phase to tailor alloy performance as we need. It is also of great interest of the slip transmission community to realize the distinguished features of dislocation transformation that two phases forming a heterophase interface with continuous slip planes can have different crystal symmetry, which is an uncharted area so far.

In this work, we have demonstrated a phenomenon, dislocation transformation, and a new Shockley partial dislocation source enabled by GSF energy surface mismatch between a high-symmetry parent phase and a low-symmetry precipitate phase. Using the $\gamma^{\prime \prime}$ phase in a $\gamma$ matrix in a widely used $\mathrm{Ni}$-base superalloy as an example, we have shown that the $\gamma^{\prime \prime}$ precipitates are able to transform a stream of CA dislocations into $C A+C B$ dislocation groups on the exit side and a single $\delta B$ Shockley partial and $\delta B / \delta A$ Shockley partial pairs on the entrance side of the precipitates. The Shockley partials generated do not originate from the dissociation of the full dislocations and the operation of the source does not depend on the stacking fault energy of the alloy. The dislocation transformation can have significant impact on deformation of $\gamma^{\prime \prime}$ and other precipitates present in the system (such as the $\gamma^{\prime}$ phase) and thus the overall mechanical properties of the alloy. With dislocation transformation, a single operating slip system results in two operating slip systems on the same slip plane when it intersects the $\gamma^{\prime \prime}$ precipitates. For slip planes that do not intersect the low symmetry precipitates, there will still be one slip system operating. This is crucial in plasticity modeling as the deformation mechanisms of the $\gamma^{\prime}$ phase are different for different dislocation groups. The Shockley partial 
source could be responsible for deformation twinning in $\gamma^{\prime \prime}$ strengthened alloys. Based on the dislocation transformation mechanism, we have proposed an alloy design strategy to tailor the $\gamma^{\prime \prime}$ precipitate microstructure by stress-aging to either suppress or promote deformation twinning. The mechanisms uncovered in this study enriches our understanding of dislocation-precipitate interaction and partial dislocation generation, which are critical for uncovering the deformation mechanisms of precipitation strengthened alloys.

\section{METHODS}

\section{Microscopic phase field model}

The microscopic phase field model has been applied successfully to study interactions between dislocations and precipitates ${ }^{9,10,18-22}$. The free energy of the system is written as follows ${ }^{16,17}$.

$F=E^{\text {cryst }}+E^{\text {grad }}+E^{\mathrm{el}}$

where $E^{\text {cryst }}$ is the crystalline energy that describes the potential energy landscape when a crystal is subjected to a general shear produced by arbitrary linear combinations of localized slips associated with all possible slip systems ${ }^{15}$. It is a periodic function reflecting the symmetry of the crystal. For a given slip plane, the crystalline energy becomes the GSF energy. $E^{\text {grad }}$ is the gradient energy that accounts for energy contributions from spatial variation of the eigenstrains of dislocations and influences the Burgers vector distribution profile in the core regions ${ }^{15}$. $E^{\mathrm{el}}$ is the elastic energy stored in the crystal caused by elastic distortion of the lattice created by dislocations, applied stress, and misfit stress. The detailed formulation of these energy terms can be found in Ref. ${ }^{16}$. All physical parameters are normalized into dimensionless values for numerical implementation ${ }^{37}$. Then the time evolution of the order parameter $\eta_{p}$ follows the time-dependent Ginzburg-Landau (TDGL) equations:

$\frac{\partial \eta_{\mathrm{p}}}{\partial \tau}=-L_{\mathrm{p}} \frac{\delta F}{\delta \eta_{\mathrm{p}}}$

where $\tau$ is the dimensionless time, $L_{p}$ is the kinetic coefficient characterizing dislocation mobility. Parameters used in the simulation are included in Supplementary Table 1.

\section{DATA AVAILABILITY}

The data that support the findings of this study are available from the corresponding author upon reasonable request.

\section{CODE AVAILABILITY}

The codes in this study are available from the authors upon reasonable request.

Received: 19 July 2021; Accepted: 27 October 2021;

Published online: 10 December 2021

\section{REFERENCES}

1. Frenkel, J. A. Zur theorie der elastizitätsgrenze und der festigkeit kristallinischer körper. Z. Für Phys. 37, 572-609 (1926).

2. Frank, F. C. \& Read, W. T. Jr Multiplication processes for slow moving dislocations. Phys. Rev. 79, 722 (1950).

3. Koehler, J. S. The nature of work-hardening. Phys. Rev. 86, 52 (1952).

4. El Kadiri, H. \& Oppedal, A. L. A crystal plasticity theory for latent hardening by glide twinning through dislocation transmutation and twin accommodation effects. J. Mech. Phys. Solids 58, 613-624 (2010).

5. Umakoshi, Y., Hagihara, K. \& Nakano, T. in Materials Science Forum, Vol. 502, 145-150 (Trans Tech Publ, 2005).

6. Yamaguchi, M. \& Umakoshi, Y. The deformation behaviour of intermetallic superlattice compounds. Prog. Mater. Sci. 34, 1-148 (1990).

7. Hagihara, K., Nakano, T. \& Umakoshi, Y. Plastic deformation behaviour and operative slip systems in Ni3Nb single crystals. Acta Mater. 48, 1469-1480 (2000).

8. Hagihara, K., Fujimoto, H., Nakano, T. \& Umakoshi, Y. Plastic deformation behavior of $\mathrm{Ni3}$ (Ti0. 7Nb0. 3) single crystals with D019 structure. Intermetallics 18, 434-440 (2010).
9. Shen, C., Mills, M. J. \& Wang, Y. Modeling dislocation dissociation and cutting of $\gamma^{\prime}$ precipitates in Ni-based superalloys by the phase field method. MRS Online Proc Libr. 753, 521 (2002).

10. Lv, D. C., McAllister, D., Mills, M. J. \& Wang, Y. Deformation mechanisms of D022 ordered intermetallic phase in superalloys. Acta Mater. 118, 350-361 (2016).

11. Hirsch, P. B. \& Kelly, A. Stacking-fault strengthening. Philos. Mag. J. Theor. Exp. Appl. Phys. 12, 881-900 (1965).

12. Ardell, A. J. Precipitation hardening. Metall. Trans. A 16, 2131-2165 (1985).

13. Nembach, E. \& Neite, G. Precipitation hardening of superalloys by ordered $\gamma$ '-particles. Prog. Mater. Sci. 29, 177-319 (1985).

14. Martin, J. W. Precipitation Hardening: Theory and Applications. (Butterworth-Heinemann, 2012).

15. Shen, C. \& Wang, Y. Phase field model of dislocation networks. Acta Mater. 51, 2595-2610 (2003).

16. Wang, Y. \& Li, J. Phase field modeling of defects and deformation. Acta Mater. $\mathbf{5 8}$ 1212-1235 (2010).

17. Wang, Y. U., Jin, Y. M., Cuitino, A. M. \& Khachaturyan, A. G. Nanoscale phase field microelasticity theory of dislocations: model and 3D simulations. Acta Mater. 49, 1847-1857 (2001).

18. Zhou, N., Shen, C., Mills, M. J., Li, J. \& Wang, Y. Modeling displacive-diffusional coupled dislocation shearing of $\gamma^{\prime}$ precipitates in Ni-base superalloys. Acta Mater. 59, 3484-3497 (2011).

19. Vorontsov, V. A., Shen, C., Wang, Y., Dye, D. \& Rae, C. M. F. Shearing of $\gamma^{\prime}$ precipitates by $a<112>$ dislocation ribbons in Ni-base superalloys: A phase field approach. Acta Mater. 58, 4110-4119 (2010).

20. Feng, L. et al. Shearing of $Y^{\prime}$ particles in Co-base and Co-Ni-base superalloys. Acta Mater. 161, 99-109 (2018).

21. Mianroodi, J. R. et al. Atomistic phase field chemomechanical modeling of dislocation-solute-precipitate interaction in Ni-Al-Co. Acta Mater. 175, 250-261 (2019).

22. Zeng, Y., Cai, X. \& Koslowski, M. Effects of the stacking fault energy fluctuations on the strengthening of alloys. Acta Mater. 164, 1-11 (2019).

23. Gao, Y., Zhou, N., Wang, D. \& Wang, Y. Pattern formation during cubic to orthorhombic martensitic transformations in shape memory alloys. Acta Mater. 68, 93-105 (2014).

24. Zhou, N. et al. Computer simulation of phase transformation and plastic deformation in IN718 superalloy: microstructural evolution during precipitation. Acta Mater. 65, 270-286 (2014).

25. Kovarik, L. et al. Microtwinning and other shearing mechanisms at intermediate temperatures in Ni-based superalloys. Prog. Mater. Sci. 54, 839-873 (2009).

26. Oblak, J. M., Paulonis, D. F. \& Duvall, D. S. Coherency strengthening in Ni base alloys hardened by DO $22 \gamma^{\prime}$ precipitates. Metall. Trans. 5, 143 (1974).

27. Merrick, H. F. Effect of heat treatment on the structure and properties of extruded P/M alloy 718. Metall. Trans. A 7, 505-514 (1976).

28. Sundararaman, M., Mukhopadhyay, P. \& Banerjee, S. Deformation behaviour of $\gamma^{\prime \prime}$ strengthened Inconel 718. Acta Metall. 36, 847-864 (1988).

29. Mahajan, S. Critique of mechanisms of formation of deformation, annealing and growth twins: Face-centered cubic metals and alloys. Scr. Mater. 68, 95-99 (2013).

30. Singh, J. B., Sundararaman, M. \& Mukhopadhyay, P. Propagation of stacking faults across domain boundaries in Ni-V and Ni-V-Nb alloys with D022 structure. Philos. Mag. A 80, 1983-2010 (2000).

31. Bayerschen, E., McBride, A. T., Reddy, B. D. \& Böhlke, T. Review on slip transmission criteria in experiments and crystal plasticity models. J. Mater. Sci. 51 2243-2258 (2016).

32. Hunter, A., Leu, B. \& Beyerlein, I. J. A review of slip transfer: applications of mesoscale techniques. J. Mater. Sci. 53, 5584-5603 (2018).

33. Werner, E. \& Prantl, W. Slip transfer across grain and phase boundaries. Acta Metall. Mater. 38, 533-537 (1990).

34. Shehadeh, M. A., Lu, G., Banerjee, S., Kioussis, N. \& Ghoniem, N. Dislocation transmission across the $\mathrm{Cu} / \mathrm{Ni}$ interface: a hybrid atomistic-continuum study. Philos. Mag. 87, 1513-1529 (2007).

35. Zeng, Y., Hunter, A., Beyerlein, I. J. \& Koslowski, M. A phase field dislocation dynamics model for a bicrystal interface system: An investigation into dislocation slip transmission across cube-on-cube interfaces. Int. J. Plast. 79, 293-313 (2016)

36. Liu, X.-Y., Capolungo, L. \& Hunter, A. Screw dislocation impingement and slip transfer at fcc-bcc semicoherent interfaces. Scr. Mater. 201, 113977 (2021).

37. Shen, $\mathrm{C}$. The fundamentals and applications of phase field method in quantitative microstructural modeling (The Ohio State University, 2004).

\section{ACKNOWLEDGEMENTS}

The authors acknowledge the financial support from NSF DMREF program under grant DMR-1922239. 


\section{AUTHOR CONTRIBUTIONS}

L.F. conducted the simulations and wrote the first draft of the manuscript. Y.W. supervised the work. All the authors contributed to the analysis of the results and discussions and were involved in writing the manuscript.

\section{COMPETING INTERESTS}

The authors declare no competing interests.

\section{ADDITIONAL INFORMATION}

Supplementary information The online version contains supplementary material available at https://doi.org/10.1038/s41524-021-00660-z.

Correspondence and requests for materials should be addressed to Yunzhi Wang.

Reprints and permission information is available at http://www.nature.com/ reprints
Publisher's note Springer Nature remains neutral with regard to jurisdictional claims in published maps and institutional affiliations.

(i) Open Access This article is licensed under a Creative Commons Attribution 4.0 International License, which permits use, sharing, adaptation, distribution and reproduction in any medium or format, as long as you give appropriate credit to the original author(s) and the source, provide a link to the Creative Commons license, and indicate if changes were made. The images or other third party material in this article are included in the article's Creative Commons license, unless indicated otherwise in a credit line to the material. If material is not included in the article's Creative Commons license and your intended use is not permitted by statutory regulation or exceeds the permitted use, you will need to obtain permission directly from the copyright holder. To view a copy of this license, visit http://creativecommons. org/licenses/by/4.0/.

(c) The Author(s) 2021 